\title{
MODELING FMRI DATA: CHALLENGES AND OPPORTUNITIES
}

\author{
Alberto Maydeu-Olivares
}

\author{
UNIVERSITY OF BARCELONA
}

\author{
GREGORY BROWN
}

UNIVERSITY OF CALIFORNIA, SAN DIEGO AND VA SAN DIEGO HEALTHCARE SYSTEM

\begin{abstract}
We offer an introduction to the five papers that make up this special section. These papers deal with a range of the methodological challenges that face researchers analyzing fMRI data-the spatial, multilevel, and longitudinal nature of the data, the sources of noise, and so on. The papers all provide analyses of data collected by a multi-site consortium, the Function Biomedical Informatics Research Network. Due to the sheer volume of data, univariate procedures are often applied, which leads to a multiple comparisons problem (since the data are necessarily multivariate). The papers in this section include interesting applications, such as a state-space model applied to these data, and conclude with a reflection on basic measurement problems in fMRI. All in all, they provide a good overview of the challenges that fMRI data present to the standard psychometric toolbox, but also to the opportunities they offer for new psychometric modeling.
\end{abstract}

Key words: network analysis, cluster analysis, three-way data, principal components analysis, independent components analysis, structural equation models, generalized linear models.

Since its introduction in the early 1990s, functional magnetic resonance imaging (fMRI) has become one of the hottest research tools in Psychology, and more generally in the Neurosciences. The purpose of fMRI is to draw conclusions about neural activation in an individual at a single time point or across time points, across individuals, or across groups. Other techniques such as event-related brain potentials (ERPs) also provide a noninvasive, high resolution way to "look at the human brain in action" (Donchin, 2006). However, an MRI has a higher spatial resolution than ERPs; it has become the method of choice for looking at the human brain in action as it provides attractive, colorful, graphical representations of brain activity.

In a typical fMRI study, data are gathered on a set of individuals while they perform a task. Most fMRI studies use the Blood Oxygenation Level Dependent (BOLD) signal as a proxy for neural activity. The BOLD signal is physiologically complex, reflecting changes in blood flow, blood volume, and oxygen metabolism at a particular location due to altered behavioral and cognitive processes. It is assumed that behavioral and cognitive processes cause neurons in a small area to become active, which increases blood flow to that area to a degree that is out of proportion to the increased metabolic demand of the neural activity. The more intense the neural activation, the larger the relative increase in blood flow will be. The greater increase in blood flow than in metabolism raises the washout rate of the paramagnetic deoxyhemoglobin molecule from the area, which in turn increases the magnetic resonance signal. The analysis strategy is to correlate the BOLD waveform with the known time course of stimulation. Brain areas engaged in the task will have a BOLD signal that is correlated with the task; brain areas that are not engaged in the task will be uncorrelated. This makes it possible to locate the brain region engaged in a particular task.

Requests for reprints should be sent to Alberto Maydeu-Olivares, Faculty of Psychology, University of Barcelona, P. Vall d'Hebron 171, 08035 Barcelona, Spain. E-mail: amaydeu@ub.edu 
In an fMRI study, the purpose may be to compare BOLD levels across populations (e.g., individuals diagnosed with schizophrenia vs. controls). Or, the purpose of the analysis may be to examine BOLD levels within individuals over time (for instance, to investigate the effects of aging). Of course, these different objectives can be combined in a single study, so that task (or treatment) effects are combined with between-population effects, and with within-individual effects. Greve, Brown, Mueller, Glover, and Liu (2012) provides a gentle introduction to how fMRI is generally analyzed at each of these levels. As is immediately apparent from Greve et al.'s article, fMRI data present a series of characteristics; many of which present a challenge to current psychometric modeling tools. fMRI data are best thought of as a 3D movie. The movie consists of shots, typically 2 seconds apart, each consisting of 122,880 data points (in the case of Greve et al.'s study) organized in a three-way array. Each of these data points is a measurement from a small box called a voxel. As described by Greve et al., typically, a generalized linear model (GLM) is applied to each voxel and the resulting parameter estimates or $t$-statistics are displayed graphically in a Beta-map or T-map, which may be used for subsequent analysis (Calhoun \& Allen, 2012).

Independent component analysis (ICA: Comon, 1994) is often used as the method of choice in the analysis of this "second level" data. It appears that principal components analysis is illsuited to analyze fMRI data, since the largest components are typically associated with motion, or other physiological noise (characteristics that are not of interest in the study). However, ICA may also be applied to the original fMRI data, and Calhoun and Allen (2012) describe a comparison of ICA modeling of the primary vs. secondary level data.

When reading Calhoun and Allen (2012) and Greve et al. (2012), psychometricians will find certain themes that have appeared in the past in the psychometric literature (and continue to reappear) such as how many components to retain (now in ICA), or how to control for the multiple comparisons used in the voxel-by-voxel GLM analysis. We believe psychometricians can offer important contributions in these areas. But many interesting questions remain open: Should not we be doing a multivoxel analysis from the onset? Should not we take into account the multilevel nature of the data? Camara, Marco-Pallarés, Münte, and Rodríguez-Fornells (2009) provided an overview of the quantitative techniques used for fMRI data and Greve et al. (2012) presented a survey of the sources of noise in these data and the challenge they represent.

Two other papers in this section address a question that might occur to many readers of the papers just mentioned. Can we apply our toolbox to these data? And if so, how? Often times fMRI studies are performed at different sites, and between-sites differences can be found due to differences in equipment, teams, and so on. Zhou, Konstorum, Duong, Tieu, Wells, Brown, Stern et al. (2012) illustrate the magnitude of this problem when aggregate results are desired and apply a multilevel model to account for these between-site differences. In the other paper, Janoos, Brown, Mórocz, and Wells (2012) use a state-space model to study working memory in schizophrenia. We hope these two papers will inspire the psychometric community to apply the methods in our toolbox to these challenging data.

And if the characteristics of fMRI data were not sufficiently challenging, the last paper in this special section forces us to think about measurement issues. Liu, Glover, Mueller, Greve, and Brown (2012) questions the validity of using the BOLD signal and asks whether it should be normalized (and if so how) or calibrated (and if so how). Liu et al. spell out some of the strong assumptions used in fMRI modeling and point out how existing attempts to improve the measurement of neural activity have yielded mixed results.

In closing, we feel that fMRI data offer interesting modeling opportunities to a wide array of psychometric researchers including, but not limited to, those interested in 3-way data, longitudinal processes, multilevel modeling, experimental design, cluster analysis, network analysis, and a long etcetera. 


\section{References}

Calhoun, V.D., \& Allen, E. (2012). Extracting intrinsic functional networks with feature-based group independent component analysis. Psychometrika. doi:10.1007/s11336-012-9291-3.

Camara, E., Marco-Pallarés, J., Münte, T.F., \& Rodríguez-Fornells, A. (2009). Neuroimaging analysis II: magnetic resonance imaging. In R.E. Millsap \& A. Maydeu-Olivares (Eds.), Handbook of quantitative methods in psychology (pp. 697-715). London: Sage.

Comon, P. (1994). Independent component analysis, a new concept? Signal Processing, 36(3), 287-314. doi:10.1016/ 0165-1684(94)90029-9.

Donchin, E. (2006). fMRI: not the only way to look at the human brain in action. Observer, 19(11).

Greve, D.N., Brown, G.G., Mueller, B.A., Glover, G., \& Liu, T.T. (2012). A survey of the sources of noise in fMRI. Psychometrika. doi:10.1007/s11336-012-9294-0.

Janoos, F., Brown, G., Mórocz, I.Á., \& Wells, W.M. (2012). State-space analysis of working memory in schizophrenia: an FBIRN study. Psychometrika. doi:10.1007/s11336-012-9300-6.

Liu, T.T., Glover, G.H., Mueller, B.A., Greve, D.N., \& Brown, G.G. (2012). An introduction to normalization and calibration methods in functional MRI. Psychometrika. doi:10.1007/s11336-012-9309-x.

Zhou, B., Konstorum, A., Duong, T., Tieu, K.H., Wells, W.M., Brown, G.G., Stern, H.S., et al. (2012). A hierarchical modeling approach to data analysis and study design in a multi-site experimental fMRI study. Psychometrika. doi:10.1007/s11336-012-9298-9.

Published Online Date: 8 MAR 2013 